Tropical Journal of Pharmaceutical Research, June 2008; 7 (2): 961-968

(c) Pharmacotherapy Group, Faculty of Pharmacy, University of Benin Benin City, Nigeria.

All rights reserved.

Research Article

Available online at http://www.tjpr.org

\title{
Synthesis, Analgesic, Anti-inflammatory and Antimicrobial Activities of Some Novel Pyrazoline Derivatives
}

\author{
SK Sahu ${ }^{\star 1}$, M Banerjee ${ }^{1}$, A Samantray ${ }^{1}$, C Behera ${ }^{1}$ and MA \\ Azam ${ }^{1}$ \\ ${ }^{1}$ University department Of Pharmaceutical Sciences, Utkal University, Bhubaneswar, Orissa, ${ }^{2}$ Department of \\ Pharmaceutical Chemistry, J. S. S., College of Pharmacy, Ootacamund-643 001, India
}

\begin{abstract}
Purpose: Microbial infections often produce pain and inflammation. Chemotherapeutic, analgesic and anti-inflammatory drugs are prescribed simultaneously in normal practice. The compound possessing all three activities is not common. The purpose of the present study was to examine whether molecular modification might result in detection of new potential antirheumatic drugs having antimicrobial activities. Method: A series of novel 4-(5'-substituted aryl-4', 5'-dihydropyrazole-3'-yl-amino) phenols 2a-f have been synthesized by treating substituted aryl- $N$-chalconyl amino phenols 1a-f with hydrazine hydrate. The starting materials were synthesized from $p$-aminoacetophenone. Their structures were confirmed by $I R,{ }^{1} H$ NMR spectral data. The synthesized compounds were investigated for analgesic, antinflammatory and antimicrobial activities.

Result: The data reported in Tables 2, 3 \& 4 shows that effect of variation in chemical structure on activity was rather unpredictable. Seldom did a particular structural modification lead to uniform alteration in activity in all tests. The substitution which appeared to be most important for high order of activity in the greatest number of test was the $p$-choloroaryl group. The introduction of $p$-nitro and $p$ hydroxy group in aryl moiety of the pyrazole analogs $2 c$ and $2 e$ produce compounds with potent analgesic, anti-inflamatory and, in a few cases, antimicrobial properties.

Conclusion: The observed increase in analgesic, anti-inflammatory and antimicrobial activities are attributed to the presence of 4- $\mathrm{NO}_{2}, 2-\mathrm{OH}$ and 4-Cl in phenyl ring at 5-position of pyrazoline ring of synthesized compounds. In some cases their activities are equal or more potent than the standard drugs.
\end{abstract}

Keywords: Pyrazole, Analgesic, Anti-inflammatory, Antibacterial activity.

*Corresponding author: Email: tutu_kh@yahoo.com, Tel: +91-9861536013 


\section{INTRODUCTION}

Pyrazole derivatives have a long history of application in agrochemicals and pharmaceutical industry as herbicides and active pharmaceuticals. The recent success of pyrazole COX-2 inhibitor has further highlighted the importance of these heterocycles in medicinal chemistry. A systematic investigation of this class of heterocyclic lead revealed that pyrazole containing pharmacoactive agents play important role in medicinal chemistry. The prevalence of pyrazole cores in biologically active molecules has stimulated the need for elegant and efficient ways to make these heterocyclic lead. The treatment of pain continues to be the subject of considerable pharmaceutical and clinical research. Microbial infections often produce pain and inflammation. Chemotherapeutic, analgesic and anti-inflammatory drugs are prescribed simultaneously in normal practice. The compound possessing all three activities is not common. It has been reported that pyrazoline possess analgesic, anti-inflammatory ${ }^{1-3}$ and antimicrobial $^{4-6}$ activities. In view of these above, an attempt has been undertaken for the synthesis of the some novel $4-\left(5^{\prime}-\right.$ substituted aryl-4', 5'-dihydropyrazole-3'-ylamino) phenols possessing potent biological activities. The synthesized compounds were tested for their possible analgesic, antiinflammatory and anti-microbial activities.

\section{EXPERIMENTAL \\ Equipment}

Melting points were determined in open capillaries and were uncorrected by melting point determining apparatus (SISCO). Purity of the compounds were checked by TLC. IR spectra $\left(\mathrm{KBr}, \mathrm{cm}^{-1}\right)$ were recorded on a JASCO FT/IR 410 spectrophotometer. ${ }^{1} \mathrm{H}$ NMR $\left(\mathrm{CDCl}_{3}\right)$ on a Bruker DPX $300-\mathrm{MHz}$ spectrometer using TMS as an internal reference (chemical shifts in $\delta \mathrm{ppm}$ ). $\mathrm{C}, \mathrm{H}$ and $\mathrm{N}$ analysis were carried out on a Euro EA (Italy) analyzer.

\section{Materials}

Pure paracetamol (ODCL,India), ciprofloxacin (Alkem,India), clotrimazole (Glenmerk,India). Methanol, Hydrazinehydrate, ethanol (all from (SD-Fine Chemical, India), Sodium Hydroxide benzaldehyde, furfuraldehyde, 4nitrobenzaldehyde, p-anisaldehyde, salicylaldehyde, 4-cholorobenzaldehyde, Carrageenan (all from Merck, Germany), carboxymethylcellulose (Sigma, India), Dimethylformamide (Aldrich), Mullar hinton agar and Sabouraud dextrose agar (Hi-Media, India).

\section{Methods}

\section{Preparation of N-(4-hydroxyphenyl)-3- phenylacrylamide (1a)}

To a mixture of $p$-hydroxyacetoaminophenone $(0.01 \mathrm{~mol})$ and benzaldehyde $(0.01 \mathrm{~mol})$ in ethanol, $2 \%$ sodium hydroxide solution $(1 \mathrm{ml})$ was added drop wise with constant stirring over a period of $30 \mathrm{~min}$. and the reaction mixture was stirred for another $10 \mathrm{~h}$ at room temperature and then refluxed for $6 \mathrm{~h}$. The excess solvent was distilled off and the solid obtained was poured into ice- cold water. The solid thus obtained was filtered, dried and recrystallised from ethanol. Compounds $\mathbf{1 b - f}$ were prepared similarly by using different arylaldehydes. Their melting points, \% yields and molecular formula are given in Table-1

1a $\left(R=-C_{6} H_{5}\right):$ m.p. $150^{\circ} \mathrm{c}$, yield: $72 \%$, $\mathrm{IR}\left(\mathrm{KBr}\right.$ in $\left.\mathrm{cm}^{-1}\right)$ : 3452 (Ar-OH str.), $3301(\mathrm{NH}$ str.), 3016 (C-H str.), 1610 (C=C str.), ${ }^{1} \mathrm{H}-$ NMR $(\delta \mathrm{ppm})\left(\mathrm{CDCl}_{3}\right), 7.1-7.8(2 \mathrm{H}, \mathrm{d}, \mathrm{CH})$, $6.11(1 \mathrm{H}, \mathrm{s}, \mathrm{N}-\mathrm{H}), 7.70(1 \mathrm{H}, \mathrm{s}, \mathrm{N}-\mathrm{H}), 5.35(1 \mathrm{H}, \mathrm{s}$, Ar-OH),6.76 - 8.00 (m, Ar-H ). Analysis $\left(\mathrm{C}_{15} \mathrm{H}_{13} \mathrm{O}_{2} \mathrm{~N}\right)$ cal(found)\%: C 75.30(75.52) H5.48(4.98) N5.85(6.21).,MS:(m/z) : 239( $\left.\mathrm{M}^{+}\right)$. 1b $\left(\mathrm{R}=-\right.$ Furyl): IR(KBr in $\left.\mathrm{cm}^{-1}\right): 3300$ (Ar$\mathrm{OH}$ str.), 3253 (NH str.), $2922\left(\mathrm{CH}_{2}\right.$ str.), 1476(C=C str.), 1137 (C-O-C str.); ${ }^{1} \mathrm{H}-\mathrm{NMR}(\delta$ ppm) $\left(\mathrm{CDCl}_{3}\right), \quad 7.13-7.21(2 \mathrm{H}, \mathrm{d}, \mathrm{CH}), \quad 6.21$ $(1 \mathrm{H}, \mathrm{s}, \mathrm{N}-\mathrm{H}), 7.38(1 \mathrm{H}, \mathrm{s}, \mathrm{N}-\mathrm{H}),, 5.65(1 \mathrm{H}, \mathrm{s}, \mathrm{Ar}-$ $\mathrm{OH}), 6.76-8.00(\mathrm{~m}, \mathrm{Ar}-\mathrm{H})$. Analysis $\left(\mathrm{C}_{13} \mathrm{H}_{11} \mathrm{O}_{3} \mathrm{~N}\right)$ ) cal(found)\%: C 68.11(68.43) H 4.84(4.49) N 6.11(5.89).,MS:(m/z): 229. 1c $\left(\mathrm{R}=\mathrm{p}-\mathrm{NO}_{2}-\mathrm{C}_{6} \mathrm{H}_{4}\right)$ :- IR( $\mathrm{KBr}$ in $\left.\mathrm{cm}^{-1}\right)$ : $3490(\mathrm{Ar}-$ $\mathrm{OH}$ str.), 3291 (NH str.), 3099 (C-H str.), 2851( $\mathrm{CH}_{2}$ str.), 1560 (C- $\mathrm{NO}_{2}$ asym. str.), 
1485(C=C str.); .), ${ }^{1} \mathrm{H}-\mathrm{NMR}(\delta \mathrm{ppm})\left(\mathrm{CDCl}_{3}\right)$, 7.1-7.8 $(2 \mathrm{H}, \mathrm{d}, \mathrm{CH}), \quad 6.13(1 \mathrm{H}, \mathrm{s}, \mathrm{N}-\mathrm{H}), \quad 7.68$ $(1 \mathrm{H}, \mathrm{s}, \mathrm{N}-\mathrm{H}), 5.35(1 \mathrm{H}, \mathrm{s}, \mathrm{Ar}-\mathrm{OH}), 6.76-8.02$ (m, Ar- $\mathrm{H}$ ) Analysis $\left(\mathrm{C}_{15} \mathrm{H}_{12} \mathrm{O}_{4} \mathrm{~N}_{2}\right)$ cal(found)\%: C 63.38(63.42) H 4.25(4.52) N 9.85(9.53). MS $:(\mathrm{m} / \mathrm{z})$ 284(M+). 1d $\left(\mathrm{R}=\mathrm{p}-\mathrm{OCH}_{3}-\mathrm{C}_{6} \mathrm{H}_{4}\right)$ $\mathrm{IR}\left(\mathrm{KBr}\right.$ in $\left.\mathrm{cm}^{-1}\right)$ : 3431(Ar-OH str.), 3211 (NH str.), $2831\left(\mathrm{CH}_{2}\right.$ str), 1493(C=C str), 1101(CO-C str); ${ }^{1} \mathrm{H}-\mathrm{NMR}(\delta \mathrm{ppm})\left(\mathrm{CDCl}_{3}\right)$ 7.1-7.8 $(2 \mathrm{H}, \mathrm{d}, \mathrm{CH}), 6.14(1 \mathrm{H}, \mathrm{s}, \mathrm{N}-\mathrm{H}), 7.52(1 \mathrm{H}, \mathrm{s}, \mathrm{N}-\mathrm{H})$, $5.35(1 \mathrm{H}, \mathrm{s}, \mathrm{Ar}-\mathrm{OH}), 6.76$ - 8.01.(m, Ar-H ). ):Analysis $\left(\mathrm{C}_{16} \mathrm{H}_{15} \mathrm{O}_{3} \mathrm{~N}\right)$ cal (found) \%: C 71.36(71.57) H 5.61(6.02) N 5.20(4.99). MS:(m/z): $269\left(\mathrm{M}^{+}\right) .1 \mathrm{e}\left(\mathrm{R}=2-\mathrm{OH}-\mathrm{C}_{6} \mathrm{H}_{4}\right):-$ $\mathrm{IR}\left(\mathrm{KBr}\right.$ in $\left.\mathrm{cm}^{-1}\right)$ : 3412 (Ar-OH str.),3208(NH str.), 2834( $\mathrm{CH}_{2}$ str. $), \quad 1505.7(\mathrm{C}=\mathrm{C}$ str. $) ;{ }^{1} \mathrm{H}-$ NMR $(\delta \mathrm{ppm})\left(\mathrm{CDCl}_{3}\right), 7.1-7.8(2 \mathrm{H}, \mathrm{d}, \mathrm{CH})$, $6.23(1 \mathrm{H}, \mathrm{s}, \mathrm{N}-\mathrm{H}), 7.48(1 \mathrm{H}, \mathrm{s}, \mathrm{N}-\mathrm{H}), 5.35(1 \mathrm{H}$, $\mathrm{s}, \mathrm{Ar}-\mathrm{OH}), 6.56-8.00(\mathrm{~m}, \mathrm{Ar}-\mathrm{H})$ Analysis $\left(\mathrm{C}_{15} \mathrm{H}_{13} \mathrm{O}_{3} \mathrm{~N}\right)$ cal(found)\%: C 70.58(70.82) H 5.13(5.34) N 5.49(5.26). MS :( m/z) 255( $\left.\mathrm{M}^{+}\right)$. $1 f\left(\mathrm{R}=\mathrm{p}-\mathrm{Cl}-\mathrm{C}_{6} \mathrm{H}_{4}\right)$ : IR $\left(\mathrm{KBr}\right.$ in $\left.\mathrm{cm}^{-1}\right): 3417(\mathrm{Ar}-$ $\mathrm{OH}$ str.), 3278(NH str.), 2932 (C-H str.), 2836 $\left(\mathrm{CH}_{2}\right.$ str. $), 742(\mathrm{C}-\mathrm{Cl}.) \cdot{ }^{1} \mathrm{H}-\mathrm{NMR}(\delta \mathrm{ppm})$ $\left(\mathrm{CDCl}_{3}\right), 7.19-7.21(2 \mathrm{H}, \mathrm{d}, \mathrm{CH}), 6.21(1 \mathrm{H}, \mathrm{s}, \mathrm{N}-$ $\mathrm{H}), 7.40(1 \mathrm{H}, \mathrm{s}, \mathrm{N}-\mathrm{H}), 5.38(1 \mathrm{H}, \mathrm{s}, \mathrm{Ar}-\mathrm{OH})$, $6.76-8.00(\mathrm{~m}, \quad$ Ar- $\mathrm{H})$. Analysis $\left(\mathrm{C}_{15} \mathrm{H}_{12} \mathrm{O}_{2} \mathrm{NCl}\right)$ cal (found) \%: C 65.82(66.12) $\mathrm{H}$ 4.42(4.63) N 5.12(5.43), MS :( $\mathrm{m} / \mathrm{z})$ $273\left(\mathrm{M}^{+}\right)$.

Preparation of 4-[(5'-phenyl-4', 5'dihydropyrazol-3'-yl) amino] phenol (2a)

A mixture of compound 1a $(0.01 \mathrm{~mol})$ and hydrazine hydrate $(0.01 \mathrm{~mol})$ in ethanol $(30 \mathrm{ml})$, were refluxed for $6 \mathrm{~h}$ on a water bath. The reaction mixture was concentrated, cooled and poured into ice-cold water. The resulting solid $\mathbf{2 a}$ was filtered, dried and recrystallised from ethanol. Compounds $\mathbf{2 b}$-f were prepared similarly. Their melting points, \% yields and molecular formula are given in Table-1. 2a( $R$ $\left.=-\mathrm{C}_{6} \mathrm{H}_{5}\right): \mathrm{IR}\left(\mathrm{KBr}\right.$ in $\left.\mathrm{cm}^{-1}\right): 3462(\mathrm{Ar}-\mathrm{OH}$ str. $)$, 3293 (NH str.), 3261(NH str.), 3022(C-H str.), 1630 (C=N str.), ${ }^{1} \mathrm{H}-\mathrm{NMR}(\delta \mathrm{ppm})\left(\mathrm{CDCl}_{3}\right)$, 2.20-2.25 (1H, t, CH), $6.15(1 \mathrm{H}, \mathrm{s}, \mathrm{N}-\mathrm{H}), \quad 7.43$ $(1 \mathrm{H}, \mathrm{d}, \mathrm{N}-\mathrm{H}), 4.71-4.86\left(2 \mathrm{H}, \mathrm{d}, \mathrm{CH}_{2}\right), 5.31$ $(1 \mathrm{H}, \mathrm{s}, \mathrm{Ar}-\mathrm{OH}), 7.31-7.65(\mathrm{~m}, \mathrm{Ar}-\mathrm{H})$. Analysis $\left(\mathrm{C}_{15} \mathrm{H}_{15} \mathrm{~N}_{3} \mathrm{O}\right)$ cal (found) \%: C 71.13(70.75) H 5.97(5.76) N 16.59(16.39). MS
:( $\mathrm{m} / \mathrm{z}):$ 253( $\left.\mathrm{M}^{+}\right) . \mathbf{2 b}$ (R=Furyl): IR ( $\mathrm{KBr}$ in $\mathrm{cm}^{-}$ 1): 3312 (Ar-OH str.), 3261 (NH str.), 3264 (NH str.), 3065 (O-H str.), $2927\left(\mathrm{CH}_{2}\right.$ str.), 1630 (C=N str.), 1464 (C=C str.), 1137 (C-O-C str.); ${ }^{1} \mathrm{H}-\mathrm{NMR}(\delta \mathrm{ppm})\left(\mathrm{CDCl}_{3}\right), 2.13-2.22(1 \mathrm{H}$, $\mathrm{t}, \mathrm{CH}), 6.24(1 \mathrm{H}, \mathrm{s}, \mathrm{N}-\mathrm{H}), 7.38(1 \mathrm{H}, \mathrm{d}, \mathrm{N}-\mathrm{H})$, 4.54- $4.61\left(2 \mathrm{H}, \mathrm{d}, \mathrm{CH}_{2}\right), 5.63(1 \mathrm{H}, \mathrm{s}, \mathrm{Ar}-\mathrm{OH})$, $6.63-7.87(\mathrm{~m}, \mathrm{Ar}-\mathrm{H})$. Analysis $\left(\mathrm{C}_{13} \mathrm{H}_{13} \mathrm{~N}_{3} \mathrm{O}_{2}\right)$ cal (found) \%: C 64.19(64.23) H 5.39(4.99) N 12.27(12.63).MS :( m/z) $243\left(M^{+}\right) .2 c(R=p-$ $\mathrm{NO}_{2}-\mathrm{C}_{6} \mathrm{H}_{4} \mathrm{IR}\left(\mathrm{KBr}\right.$ in cm $\left.\mathrm{cm}^{-1}\right): 3497$ (Ar-OH str.), 3298 (NH str.), 3241 (NH str.), 3073 (C-H str.), $2832\left(\mathrm{CH}_{2}\right.$ str.), 1634 ( $\mathrm{C}=\mathrm{N}$ str.), 1570 (C$\mathrm{NO}_{2}$ asym. str.), 1489 ( $\mathrm{C}=\mathrm{C}$ str. $) ;{ }^{1} \mathrm{H}-\mathrm{NMR}(\delta$ ppm) $\quad\left(\mathrm{CDCl}_{3}\right), 2.22-2.24 \quad(1 \mathrm{H}, \quad \mathrm{t}$, $\mathrm{CH}), / 6.11(1 \mathrm{H}, \mathrm{s}, \mathrm{N}-\mathrm{H}), 7.68(1 \mathrm{H}, \mathrm{d}, \mathrm{N}-\mathrm{H}), 4.11-$ $4.16\left(2 \mathrm{H}, \mathrm{d}, \mathrm{CH}_{2}\right), 5.28(1 \mathrm{H}, \mathrm{s}, \mathrm{Ar}-\mathrm{OH}), 6.78-$ $8.24(\mathrm{~m}$, Ar-H $)$. ):- Analysis $\left(\mathrm{C}_{15} \mathrm{H}_{14} \mathrm{~N}_{4} \mathrm{O}_{3}\right)$ cal(found)\%: C $60.40(60.49) \mathrm{H} 4.73(4.87) \mathrm{N}$ 18.78 (18.43). MS :( m/z) 298( $\left.M^{+}\right) .2 d(R=p-$ $\left.\mathrm{OCH}_{3}-\mathrm{C}_{6} \mathrm{H}_{4}\right)$ :- IR(KBr in cm $\left.\mathrm{cm}^{-1}\right): 3431$ (Ar-OH str.), 3207 ( $\mathrm{NH}$ str.), $2843\left(\mathrm{CH}_{2} \mathrm{str}\right), 1613$ (C=N str), 1499 (C=C str), 1093 (C-O-C str); ${ }^{1} \mathrm{H}-\mathrm{NMR}(\delta \mathrm{ppm})\left(\mathrm{CDCl}_{3}\right), 2.1-2.4(1 \mathrm{H}, \mathrm{t}, \mathrm{CH})$, 6.17(1H,s, N-H), $7.52(1 \mathrm{H}, \mathrm{d}, \mathrm{N}-\mathrm{H}), 4.36-4.41$ $\left(2 \mathrm{H}, \mathrm{d}, \mathrm{CH}_{2}\right), 5.47(1 \mathrm{H}, \mathrm{s}, \mathrm{Ar}-\mathrm{OH}), 6.71-8.11$ ( $\mathrm{m}, \mathrm{Ar}-\mathrm{H})$. Analysis $\left(\mathrm{C}_{16} \mathrm{H}_{17} \mathrm{~N}_{3} \mathrm{O}_{2}\right.$. $)$ cal (found) \%: C 67.83 (68.01) H 6.05 (5.88) N 14.83 (14.72). $\quad M S:(m / z) \quad 283\left(M^{+}\right) .2 e \quad(R=2-O H-$ $\left.\mathrm{C}_{6} \mathrm{H}_{4}\right)$ :- $\mathrm{IR}\left(\mathrm{KBr}\right.$ in $\left.\mathrm{cm}^{-1}\right)$ : 3409 (Ar-OH str.),3202 (NH str.), $2834\left(\mathrm{CH}_{2}\right.$ str.), 1638 $(\mathrm{C}=\mathrm{N}$ str. $), 1500(\mathrm{C}=\mathrm{C}$ str. $) ;{ }^{1} \mathrm{H}-\mathrm{NMR}(\delta \mathrm{ppm})$ $\left(\mathrm{CDCl}_{3}\right), 2.12-2.6(1 \mathrm{H}, \mathrm{t}, \mathrm{CH}), 6.26(1 \mathrm{H}, \mathrm{s}, \mathrm{N}-\mathrm{H})$, $7.48(1 \mathrm{H}, \mathrm{d}, \mathrm{N}-\mathrm{H}), 4.15-4.18\left(2 \mathrm{H}, \mathrm{d}, \mathrm{CH}_{2}\right)$, $5.34(1 \mathrm{H}, \mathrm{s}, \mathrm{Ar}-\mathrm{OH}), 6.68-8.13(\mathrm{~m}, \mathrm{Ar}-\mathrm{H})$. Analysis $\left(\mathrm{C}_{15} \mathrm{H}_{15} \mathrm{~N}_{3} \mathrm{O}_{2}\right)$ cal (found) \%: C 66.96(66.64) H 5.61(5.47), N 15.60 (15.34). MS :( m/z) $269\left(\mathrm{M}^{+}\right) .2 \mathrm{f}\left(\mathrm{R}=\mathrm{p}-\mathrm{Cl}-\mathrm{C}_{6} \mathrm{H}_{4}\right): \mathrm{IR}(\mathrm{KBr}$ in $\mathrm{cm}^{-1}$ ) : 3411 (Ar-OH str.), 3271 (NH str.), 2922 (C-H str.), 2922 ( $\mathrm{CH}_{2}$ str.), 1024 (C=N str.), 742 (C-Cl str.), ${ }^{1} \mathrm{H}-\mathrm{NMR}(\delta \mathrm{ppm})$ $\left(\mathrm{CDCl}_{3}\right), 2.21-2.23(1 \mathrm{H}, \mathrm{t}, \mathrm{CH}), 6.24(1 \mathrm{H}, \mathrm{s}, \mathrm{N}-$ $\mathrm{H}), 7.43(1 \mathrm{H}, \mathrm{d}, \mathrm{N}-\mathrm{H}), 4.23-4.28\left(2 \mathrm{H}, \mathrm{d}, \mathrm{CH}_{2}\right)$, $5.46(1 \mathrm{H}, \mathrm{s}, \mathrm{Ar}-\mathrm{OH}), 6.82-8.16(\mathrm{~m}, \mathrm{Ar}-\mathrm{H})$. Analysis $\left(\mathrm{C}_{15} \mathrm{H}_{14} \mathrm{~N}_{3} \mathrm{OCl}\right)$ cal (found) \%: C 62.61(62.78) H 4.90(4.92) N 14.60(14.29), MS $:(\mathrm{m} / \mathrm{z}) 287\left(\mathrm{M}^{+}\right)$.

\section{Animals}

Wistar albino mice (20-30 g) and Swiss albino rats $(100-140 \mathrm{~g})$ of either sex were selected 
for the experiments. Animals were allowed to be acclimatise for a period of 2 weeks in our laboratory environment prior to the study. Animals were housed in polypropylene cages (4 animals per cage), maintained under standard laboratory conditions (i.e. 12:12 hour light and dark sequence; at an ambient temperature of $25 \pm 2^{\circ} \mathrm{C}$; $35-60 \%$ humidity); the animals were fed with standard rat pellet diet (Hindustan Liver Ltd.Mumbai) and water ad libitum. The principles of Laboratory Animal Care (NIH, 1985) were followed and instructions given by our institutional animal ethical committee were maintained throughout the experiment.

\section{Analgesic activity}

The analgesic activity was determined by tail flick method ${ }^{7}$. Wistar albino mice of either sex $(20-30 \mathrm{~g})$ in the groups of six animals each were selected by random sampling technique. Paracetamol at a dose level of $100 \mathrm{mg} / \mathrm{kg}$ was administered as a reference drug for comparison. The test compounds at dose level of $100 \mathrm{mg} / \mathrm{kg}$ were administered orally by intragastric tube. The animals were held in position by a suitable restrained with the tail extending out and the tail (up to $5 \mathrm{~cm}$ ) was then dipped in a beaker of water maintained at $55 \pm 5{ }^{\circ} \mathrm{C}$. The time in seconds taken to withdraw the tail clearly out of water was taken as the reaction time. The reading was recorded at $30,60,120$ and $180 \mathrm{~min}$. after administration of compounds. A cut off point of $10 \mathrm{sec}$. was observed to prevent the tail damage. The results are presented in Table-2.

\section{Anti-inflammatory activity}

The anti-inflammatory activity was determined by carrageenan-induced rat paw oedema method ${ }^{7}$ in abino rats $(n=6)$ of eiher sex (100$140 \mathrm{~g})$. Rats were selected by random sampling technique. Paracetamol $(100 \mathrm{mg} / \mathrm{kg})$ was administered as a reference drug. The test compounds were administered at dose level of $100 \mathrm{mg} / \mathrm{kg}$ orally $30 \mathrm{~min}$. prior to the administration of carrageenan in the right hind paw of the rats. The paw thickness was measured using vernier callipers at 30, 60, 120 and 180 min. after carrageenan administration. The results are presented in Table-3.

\section{Antimicrobial activity}

In vitro antimicrobial study was carried on Muller hinton agar (Hi-media) plates $\left(37^{\circ} \mathrm{C}\right.$, $24 \mathrm{~h}$ ) by agar diffusion cup plate method ${ }^{8}$. All the compounds were screened for antimicrobial activity at $100 \mu \mathrm{g} / \mathrm{ml}$ concentration against the following bacterial strains: Staphylococcus aureus, Staphylococcus feacalis, Escherichia coli, and Salmonella typhi. Antifungal activity was tested on Sabouraud dextrose agar (Himedia) plates $\left(26{ }^{\circ} \mathrm{C}, 48-72 \mathrm{~h}\right)$ by cup plate method ${ }^{8}$ against Candida albicans and Aspergillus niger at the concentration level of $100 \mu \mathrm{g} / \mathrm{ml}$, Ciprofloxacin and Clotrimazole were used as a standards for comparison of antibacterial and antifungal activity under the similar conditions. DMF was used as a solvent control for both antibacterial and anti fungal activities. The results are presented in Table-4.

\section{Statistical analysis}

Data were analyzed by one -way ANOVA followed by Dunnett's $t$-test using computerized Graph Pad Instat version 3.05 (Graph Pad software, U.S.A.).

\section{RESULTS}

Biological results are reported in Table 2, 3 and 4, which also records the effects of the standard drug included for comparison, Series of compound are prepared in this study exhibited significant pharmacological properties in different biological models. The general pattern of pharmacological activity encountered in this synthesized compounds was seen mainly in their effect on pain perception and local inflamation. However, there was a small, well defined antimicrobial activity range associated with many of these compounds. Considerable variation of these effects were seen with each structural change, varying from agents that had less activity to those with high potency, and significant changes in potency resulted even from minor change in chemical structure as shown in Table2,3 and 4. 
Table 1: Characterization data of compounds 1a-f and 2a-f<smiles>Oc1ccc(NC2=NNC(P)C2)cc1</smiles>

\begin{tabular}{cllrl}
\hline Compound & $(\mathrm{R})$ & Mol. Form. & M.P. $\left({ }^{0} \mathrm{C}\right)$ & Yield (\%) \\
\hline $1 \mathrm{a}$ & $-\mathrm{C}_{6} \mathrm{H}_{5}$ & $\mathrm{C}_{15} \mathrm{H}_{13} \mathrm{O}_{2} \mathrm{~N}$ & $148-150$ & 72 \\
$1 \mathrm{~b}$ & $-2-\mathrm{furyl}_{13}$ & $\mathrm{C}_{13} \mathrm{H}_{11} \mathrm{O}_{3} \mathrm{~N}$ & $160-162$ & 63 \\
$1 \mathrm{c}$ & $-4-\mathrm{NO}_{2}-\mathrm{C}_{6} \mathrm{H}_{4}$ & $\mathrm{C}_{15} \mathrm{H}_{12} \mathrm{O}_{4} \mathrm{~N}_{2}$ & $108-110$ & 81 \\
$1 \mathrm{~d}$ & $-4-\mathrm{OCH}_{3}-\mathrm{C}_{6} \mathrm{H}_{4}$ & $\mathrm{C}_{16} \mathrm{H}_{15} \mathrm{O}_{3} \mathrm{~N}$ & $152-154$ & 74 \\
$1 \mathrm{e}$ & $-2-\mathrm{OH}_{-}-\mathrm{C}_{6} \mathrm{H}_{4}$ & $\mathrm{C}_{15} \mathrm{H}_{13} \mathrm{O}_{3} \mathrm{~N}$ & $142-144$ & 72 \\
$1 \mathrm{f}$ & $-4-\mathrm{Cl}-\mathrm{C}_{6} \mathrm{H}_{4}$ & $\mathrm{C}_{15} \mathrm{H}_{12} \mathrm{O}_{2} \mathrm{NCl}$ & $150-152$ & 75 \\
$2 \mathrm{a}$ & $-\mathrm{C}_{6} \mathrm{H}_{5}$ & $\mathrm{C}_{15} \mathrm{H}_{15} \mathrm{~N}_{3} \mathrm{O}$ & $161-163$ & 62 \\
$2 \mathrm{~b}$ & $-2-\mathrm{furyl}_{2}$ & $\mathrm{C}_{13} \mathrm{H}_{13} \mathrm{~N}_{3} \mathrm{O}_{2}$ & $159-161$ & 73 \\
$2 \mathrm{c}$ & $-4-\mathrm{NO}_{2}-\mathrm{C}_{6} \mathrm{H}_{4}$ & $\mathrm{C}_{15} \mathrm{H}_{14} \mathrm{~N}_{4} \mathrm{O}_{3}$ & $182-184$ & 69 \\
$2 \mathrm{~d}$ & $-4-\mathrm{OCH}_{3}-\mathrm{C}_{6} \mathrm{H}_{4}$ & $\mathrm{C}_{16} \mathrm{H}_{17} \mathrm{~N}_{3} \mathrm{O}_{2}$ & $143-145$ & 72 \\
$2 \mathrm{e}$ & $-2-\mathrm{OH}-\mathrm{C}_{6} \mathrm{H}_{4}$ & $\mathrm{C}_{15} \mathrm{H}_{15} \mathrm{~N}_{3} \mathrm{O}_{2}$ & $164-166$ & 66 \\
$2 \mathrm{f}$ & $-4-\mathrm{Cl}-\mathrm{C}_{6} \mathrm{H}_{4}$ & $\mathrm{C}_{15} \mathrm{H}_{14} \mathrm{~N}_{3} \mathrm{OCl}$ & $190-192$ & 78 \\
\hline
\end{tabular}

Table 2: Analgesic activity (tail flick method) of compounds 2a-f

\begin{tabular}{|c|c|c|c|c|c|}
\hline \multirow[t]{2}{*}{ Compd. } & \multirow{2}{*}{$\begin{array}{l}\text { Dose } \\
\mathrm{mg} / \mathrm{kg}\end{array}$} & \multicolumn{4}{|c|}{ Percentage of analgesic activity } \\
\hline & & $30 \mathrm{~min}$. & 1 hour & 2 hour & 3 hour \\
\hline $2 a$ & 100 & $27 \pm 0.12^{*}$ & $30 \pm 0.23^{*}$ & $35 \pm 0.43^{*}$ & $30 \pm 0.11^{\star}$ \\
\hline $2 b$ & 100 & $33 \pm 0.25^{\star}$ & $42 \pm 0.09^{* *}$ & $44 \pm 0.40^{*}$ & $38 \pm 0.31^{*}$ \\
\hline $2 c$ & 100 & $38 \pm 0.54^{* *}$ & $43 \pm 0.23^{* *}$ & $47 \pm 0.43^{* *}$ & $38 \pm 0.29^{*}$ \\
\hline $2 d$ & 100 & $44 \pm 0.23^{*}$ & $53 \pm 0.29^{* *}$ & $58 \pm 0.33^{*}$ & $45 \pm 0.36$ \\
\hline $2 e$ & 100 & $36 \pm 0.32^{*}$ & $43 \pm 0.36^{* *}$ & $47 \pm 0.38^{* *}$ & $38 \pm 0.42^{* *}$ \\
\hline $2 f$ & 100 & $42 \pm 0.23^{* *}$ & $45 \pm 0.73^{\star *}$ & $50 \pm 0.87^{* \star}$ & $38 \pm 0.65 *$ \\
\hline Paracetamol & 100 & $38 \pm 0.42^{* *}$ & $47 \pm 0.82^{* *}$ & $52 \pm 0.71^{* *}$ & $33 \pm 0.31^{*}$ \\
\hline Control & & $3 \pm 0.26$ & $6 \pm 0.44$ & $4 \pm 0.57$ & $4 \pm 0.91$ \\
\hline
\end{tabular}

Analgesic Activity: Some of the compounds in this series exhibited activity in experimental models used. The particular interests are the results obtained in the Glassman's procedure which utilizes selective inhibition of inflammatory pain as a creation for antiinflammatory drugs. When the structure of this synthesized compound is compared, it would appear that replacement in $\mathrm{R}$ with a $\mathrm{p}$-nitro, $\mathrm{p}$ - methoxy and p-choloro aryl groups (2c, 2e \&2f) showed promising analgesic activity.

Anti-inflammatory Activity: A number of agents caused marked reduction of the carrageenan induced edema of the rat foot, however, with exception of compounds $2 f(R=$ p-Nitro phenyl). In this test also only analogs with a p-Methoxy phenyl group in $R(2 d)$ showed equal to that exhibited by the standard paracetamol .Compounds $2 f$, in 
Table 3: Anti-inflammatory activity (carrageenan induced rat paw oedema method) of compounds 2a-f.

\begin{tabular}{|c|c|c|c|c|c|}
\hline \multirow[t]{2}{*}{ Compd. } & \multirow{2}{*}{$\begin{array}{l}\text { Dose } \\
\mathrm{mg} / \mathrm{kg}\end{array}$} & \multicolumn{3}{|c|}{ Percentage inhibition } & \multirow[b]{2}{*}{3 hour } \\
\hline & & $30 \mathrm{~min}$. & 1 hour & 2 hour & \\
\hline $2 a$ & 100 & $26 \pm 0.10$ * & $32 \pm 0.62$ * & $39 \pm 0.10^{*}$ & $33 \pm 0.07^{*}$ \\
\hline $2 b$ & 100 & $28 \pm 0.19^{*}$ & $37 \pm 0.17^{*}$ & $43 \pm 0.78$ * & $36 \pm 0.17^{*}$ \\
\hline $2 \mathrm{c}$ & 100 & $27 \pm 0.41^{\star *}$ & $33 \pm 0.81$ * & $38 \pm 0.67^{*}$ & $29 \pm 0.24$ * \\
\hline $2 d$ & 100 & $26 \pm 0.40^{*}$ & $32 \pm 0.36$ ** & $35 \pm 0.96$ ** & $27 \pm 0.66$ ** \\
\hline $2 e$ & 100 & $28 \pm 0.27^{\star *}$ & $35 \pm 0.49^{\star *}$ & $41 \pm 0.11^{*}$ & $32 \pm 0.53^{*}$ \\
\hline $2 f$ & 100 & $29 \pm 0.78^{* *}$ & $33 \pm 0.27^{* *}$ & $34 \pm 0.42^{* *}$ & $27 \pm 0.62$ ** \\
\hline Control & & $5.11 \pm 0.28$ & $6.13 \pm 0.26$ & $5.68 \pm 0.36$ & $3.30 \pm 0.91$ \\
\hline Paracetamol & 100 & $26 \pm 0.29^{* *}$ & $30 \pm 0.22^{* *}$ & $34 \pm 0.91^{* *}$ & $28 \pm 0.62$ ** \\
\hline
\end{tabular}

Table 4: Antibacterial and antifungal activity of compounds 2(a-f)

\begin{tabular}{cccccccc}
\hline Compd. & Conc. $(\mu \mathrm{g} / \mathrm{ml})$ & \multicolumn{2}{c}{ Zone } & \multicolumn{2}{c}{ of inhibition $(\mathrm{mm})$} & & \\
& & S. $a$ & S. $f$ & E. $c$ & S. $t$ & C. $a$ & A. $n$ \\
\hline $2 \mathrm{a}$ & 100 & 14 & 16 & 16 & 12 & 13 & 13 \\
$2 \mathrm{~b}$ & 100 & 13 & 12 & 15 & 11 & 12 & 11 \\
$2 \mathrm{c}$ & 100 & 18 & 20 & 21 & 19 & 17 & 19 \\
$2 \mathrm{~d}$ & 100 & 16 & 15 & 17 & 14 & 14 & 13 \\
$2 \mathrm{e}$ & 100 & 21 & 16 & 17 & 19 & 20 & 17 \\
$2 \mathrm{f}$ & 100 & 18 & 18 & 19 & 18 & 21 & 24 \\
Ciprofloxacine & 10 & 29 & 31 & 32 & 26 & - & - \\
Clotrimazole & 20 & - & - & - & - & 28 & 27 \\
\hline
\end{tabular}

${ }^{*}$ Average of three readings

S. a = Staphylococcus aureus; S.f = Staphylococcus faecalis; $E . \quad c=$ Escherichia coli; $S . t=$ Salmonella typhi; $C$. Candida albicans; $A$. $n=$ Aspergillus niger

addition to being the most potent agents of this series against rat-foot inflammation, were also found to be among the most active analgesic when assayed in Glassman's analgesic model.

Antimicrobial Activity: The in-vitro antimicrobial activity of compounds (2a-f) were determined by agar cup plate method, The results of which are summarized in Table 4.The antimicrobial data in table 4 clearly showed that the halogen, nitro \& hydroxyl phenyl groups is by far the most active substituted $R$ group. The methoxy group generally confers week antimicrobial activity. Phenyl and Furyl substitution are weakly active to inactive among the synthesized compounds. Compounds 2c, $2 \mathrm{e}$ and $2 \mathrm{f}$ showed good activity against $S$. aureous and S. typhi. The compound 2c\& $2 f$ exhibit promising activity against $C$. albicans and $A$. niger. However, the tested compounds were less active in comparison to Ciprofloxacin and Clotrimazole (standard Drugs). 


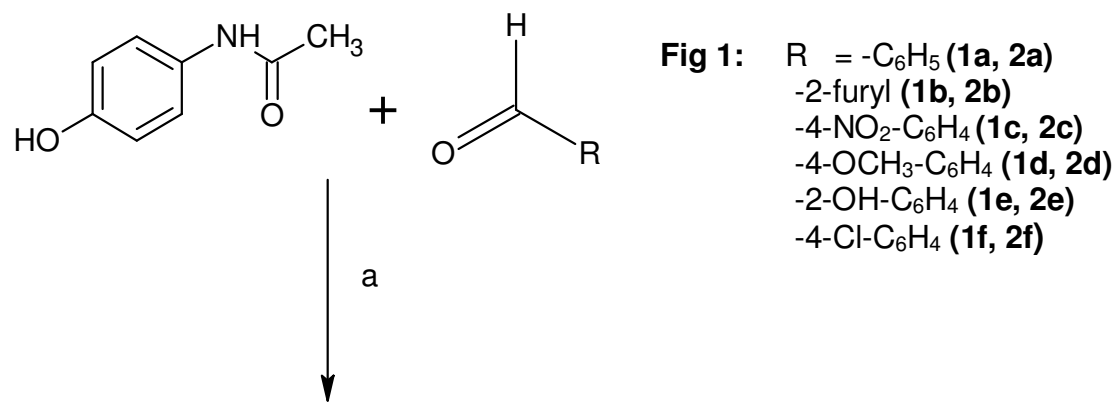<smiles>[R]/C=C\C(=O)Nc1ccc(O)cc1</smiles>

\section{DISCUSSION}

The purpose of the present study was to examine whether molecular modification might result in detection of new potential antirheumatic drugs. A series of compounds were prepared and assayed in a variety of biological test for analgesic, anti-inflammatory and antimicrobial activity. The data reported in Table 2, 3 \& 4 shows that effect of variation in chemical structure on activity was rather unpredictable. Seldom did a particular structural modification lead to uniform alteration in activity in all tests. However some point of interest did emerge and a few generalizations can be made. The substitution which appeared to be most important for high order of activity in the greatest number of test was the p-choloroaryl group. The introduction of Para nitro and p-hydroxy group in aryl moiety of the pyrazole analogs $2 \mathrm{c}$ and $2 \mathrm{e}$ produce compounds with potent analgesic, anti-inflamatory and, in a few cases, antimicrobial properties.

\section{CONCLUSION}

In conclusion, the results of this investigation revealed that the observed increase in analgesic, anti-inflammatory and antimicrobial activities are attributed to the presence of 4$\mathrm{NO}_{2}, 2-\mathrm{OH}$ and $4-\mathrm{Cl}$ in phenyl ring at 5position of pyrazoline ring of synthesized compounds. .Obviously, the comparative evaluation of active compounds will required further studies; the data reported in this article may be helpful guide for the medicinal chemist who are working in this area. 


\section{ACKNOWLEDGEMENT}

The authors would like to express their gratitude and thanks to the Head, University Dept. of Pharmaceutical Sciences, Utkal University and J. S. S., College of Pharmacy, Ootacamund for necessary facilities for this research work.

\section{REFERENCES}

1. Amir M, Kumar S. Synthesis and Anti-inflammatory, Analgesic, Ulcerogenic and Lipid Peroxidation Activities of 3, 5-Dimethyl Pyrazoles, 3-Methyl Pyrazol-5-Ones and 3, 5-Disubstituted Pyrazolines. Indian J. Chem 2005; 44B: 25322537.

2. Zelenin KN, Bezhan IP, Pastushenkov LV, Gromova EG, Lesiovskaja EE, Chakchir BA, Melnikova LF. Anti-inflammatory activity of 2-acyl-5(3)hydroxytetrahydro-1H-pyrazole derivatives. Arzneimittelforschung 1999; 49(10):_843-8.

3. Adnan AB, Hayam MAA, Aida AG. Novel Pyrazole Derivatives as Potential Promising Antiinflammatory Antimicrobial Agents. Archiv der Pharmazie 2005; 338: 167-174.
4. Susant SK, Mrityunjay B, Sagar MK, Raj MK, Prasanna PK and Prafulla MK. Synthesis, Partition Coefficient and Antibacterial Activity of 3-Phenyl (Substituted )-6-Aryl-2(1H)-Cis3',3a'-Dihydrospiro [3-H-Indole-3,5'Pyrazolo(3',4'-d)-Thiazolo-2-(1H)-ones]. Acta Poloniae Pharmaceutica-Drug Research 2007; 64: 121-126.

5. Akihiko T, Yoshihiro O, Keiko O, Hideo T, Motoji K, Masaaki $W$ and Jun-ichi $Y$. Synthesis and antibacterial activity of a novel series of DNA gyrase inhibitors: 5-[(E)-2-arylvinyl]pyrazoles. Bioorganic \& Medicinal Chemistry Letters 2005; 15: 4299-4303.

6. Goda FE, Maarouf AR, Bendary ER. Synthesis and Antimicrobial Evaluation of New Isoxazole and Pyrazole Derivaties. S. Pharm. J. 2003; 11: 111-117.

7. Kar DM, Sahu SK and Misro PK. Pharmacological activities of 1-Acetyl -3 (3-Methylanilino-2Hydroxypropyl Oximino) Indole-2, 3-Dione. INDIAN DRUG 2003; 40(5): 261-266.

8. Anonymous, British Pharmacopoeia, Voll II, H. M. S. O. Publication Centre, London 1988; A205. 